\title{
INDEX OF ISSUE 1
}

A

Ad hoc arbitration, China, 43-44, 52-53

Arbitration Act, 44

harmonisation, 49-52

Hengqin Pilot Free Trade Zone, 47-48

Internet Arbitration Alliance and Docking Rules, 48-49

pro-institutional stance, 45

SPC's Opinion on Pilot Free Trade Zones, $45-47$

Amended Model Law, See Singapore

Convention on Mediation and

Amended Model Law

Applicable law (lex loci arbitri), 35, 37-39

Arbitral tribunal, irregular composition, 34-39

Arbitration Act, 43, 44

Arbitration and Conciliation Act, 17-19

Arbitration Law of the People's Republic of China, 13

Arbitrator bias, 39-41

Arbitrator's impartiality, 31-34

Article V(1)(d) of the New York Convention, 30-32, 41

arbitral tribunal, irregular composition, 34-39

arbitrator bias, $39-41$

structure, 32-34

B

Belt and Road Initiative (BRI), 15

\section{C}

CCPIT/CCOIC Mediation Centre, 12, 14, 15

China

ad hoc arbitration (See Ad hoc arbitration, China)

dual-track system, 52

Internet Arbitration Alliance and Docking

Rules, 48-49

mediation, 11-16
China International Economic Trade

Arbitration Centre (CIETAC), 45

Civil Procedure Law of the People's Republic of China, 13, 15

Commercial Courts Act 2105, 17

Commercial Reservation, 49

Convention on the Settlement of Investment Disputes between States and Nationals of Other States (ICSID Convention), $50-51$

D

Decision-making process, 33

Docking Rules, 48-49

Dual-track system, 52

$\mathrm{E}$

eBRAM.hk, 22

G

Global Pound Conference Series, 3

Guangzhou Ocean Shipping v. Marships of Connecticut (1990), 50

Guidance on Further Advancing Mediation System to Resolve Disputes, 15

$\mathrm{H}$

Harmonisation, 49-52

Hengqin Pilot Free Trade Zone, 47-48

Hong Kong mediation, 20-23

I

ICSID Convention, 50-51

India mediation, 16-19

International Commercial Expert Committee, $15-16$

International Institute for Conflict

Prevention and Resolution, 1-2

Internet Arbitration Alliance, 48-49 
M

Maxwell Chambers, 23

Model Law on International Commercial Mediation, 4, 5, 19, 37, 43

N

New York Convention, 9, See also Article V(1)

(d) of the New York Convention

$\mathrm{P}$

Pilot free trade zones (PFTZ), 44-47

Pro-institutional stance, 45

$\mathrm{R}$

Reciprocity Reservation, 49

S

Salem Advocate Bar Assn. (II) v. Union of India, 18

Singapore Convention on Mediation and Amended Model Law, 2-6, 14-19, 22, 24-27

China, 11-16

defences to enforcement, $8-11$

form requirements, 7

Hong Kong, 20-23

India, 16-19

Singapore International Mediation Centre (SIMC), 15, 23-24

Singapore International Mediation Institute (SIMI), 23-24
Structured process, 24

Supreme Court of Germany, 37

Supreme People's Court (SPC), 44

model case, 45

Opinion on Pilot Free Trade Zones, 45-47

$\mathrm{T}$

Three-designation arbitration, 49

$\mathrm{U}$

UNCITRALWorking Group II, See Working Group

United Nations Commission on International Trade Law (UNCITRAL)

Convention on International Settlement Agreements Resulting from Mediation, 4, 5, 19-21

$\mathrm{V}$

Venire contra factum proprium,

$$
\text { 34-35 }
$$

Vikram Bakshi v. Ms Sonia Khosla, 16

W

Waiver, 34-39

Working Group, 4-6, 9, 21-23, 27

Y

Yisheng v. Invista, 45 


\section{INDEX OF ISSUE 2}

A

A. Ayyasamy v. A. Paramasivam and Others, 163,165

Abu Dhabi arbitration, 122-123

Abuse, 74-76

Abu Zahra, Muhammad, 124

Active remedy, 88-89, 102

Additional Award, 147

Alternative Delay Costs Claim, 146

Al Ziblaya, 124

Ameet Lalchand Shah and Ors. v. Rishabh Enterprises and Ors., 165

Amiable composition, 65-69, 72

Applicable law, 130-132

Arabian American Oil Company (Aramco), 120

Aramco case, 119-120, 132

applicable law, 130-132

arbitration, 120-121

foreign investors, 129-130

intention of parties, $126-128$

Islamic law

choice-of-law and, 121-123

lack of knowledge, 128

relevance of, 123-126

Arbitral legal order, 97

Arbitration

and amiable composition, 65-69

ex aequo et bono in, 71-73

Arbitration Act (AA), 112

Arbitration agreement, 114-115

Asquith, Lord, 122-123, 130-131

B

Belgian Judicial Code, 108

Belgium, 108

Bělohlávek, A. J., 83

Berger, K. P., 81

Bertrand, E., 73, 76

Born, G., 72, 79
Bruder, A. E., 82

Bucknill, A., 128

Burckhardt, P., 67

C

Canada, 90

Chauvinism, 130

Choice-of-law, 121-123

Coderre v. Coderre, 79

Commercial Arbitration Act 2010 (NSW) (Act), 135

Commercial Arbitration Act 2011 (Vic) (CAA

(Vic)), 142, 145, 147-148

Commercial Arbitration Act 2012 (WA) (2012 Act), 155

Commercial Arbitration Act 2013 (Qld) (CAA (Qld)), 139, 146

Concession agreement, 129, 130

Concession rights, 121

Concessions for exploitation (Iqta' al-Isteglal), 123, 124

Concessions for possession (Iqta' al-Tamlik), 123

Contract, 79-82

Contractual provisions, $115-116$

Court's power to set aside an arbitral award, 91

Cross-Appellants, 137-138

D

Dallah Real Estate and Tourism Holding

Company v. The Ministry of Religious

Affairs, Government of Pakistan, 104-105

December Award, 146

Decision-making, 64, 66, 72, 76, 118

Delaume, G., 131-132

Della Valle, M., 67, 70

Dispute resolution mechanism, 113

Double judicial control, 104-105 
Dual-track legislative system, 112

Dutch law, 84

E

Enforcement proceedings, 100-106

English Arbitration Act 1996, 66

Equity, 70-71, 77

European Convention of Human Rights (ECHR), 103-104

European law, 127

Ex aequo et bono, 63-65, 86 abusive exercise, 74-76

and amiable composition, 65-69

in commercial arbitration, 71-73

contract, 79-82

and equity, 70-71

implication, 84-86

public order, mandatory rules to, $83-84$

trade usages, 82-83

unpredictability, 76-78

utility of, 73-74

\section{F}

Fairness, arbitral award, 73-74

Federal Arbitration Act, 103

Fiona Trust \& Holding Corporation v. Privalov (2007), 135-136

Flexibility, 64, 73, 74, 85, 92, 94, 95, 99, 113, 117,126

Foreign investors, 129-130

France, 96-98, 107-108

Free On Board (FOB), 121

French Code of Civil Procedure, 96

French law, 107

Full Federal Court, 135-136

\section{G}

'General principles of law,' 131

Geneva, 98

Gulf oil case, 122

\section{$\mathrm{H}$}

Hancock Prospecting Pty Ltd v. Rinehart, 140

Hilgard, M., 82

Hilmarton Limited v. Omnium de Traitement et de Valorisation, 102-103

Hima, 125

Hoeft v. MVL Group, 92

Hurdsman v. Ekactrm Solutions Pty Ltd, 138-139
Hyundai Engineering \& Steel Industries Co Ltd v. Two Ways Constructions Pty Ltd, 150-151

I

ICC arbitral awards, 73-74

Imam Malik, 125

In re Chromalloy Aeroservices Inc. v. Arab Republic of Egypt, 103

Intention of parties, 126-128

International Arbitration Act 1974 (Cth), 139, 140

International Arbitration Act (IAA), 88, 101, 112,114

International arbitration and foreign parties, 112-113

International Chamber of Commerce (ICC Rules), 95

Internationalization, 131-132

Internationalization of state contracts theory, 121-122

Investment arbitration regime, 132

IPCO (Nigeria) Ltd v. Nigerian National Petroleum Corp, 149

Iqta', 123-125

Islamic judicial system, 128

Islamic law, 119

choice-of-law and, 121-123

lack of knowledge, 128

relevance of, 123-126

J

Jurisdictions, set-aside proceedings, 91-97

K

Kerr, M., 69

Kingdom of Saudi Arabia v Arabian American Oil Company (ARAMCO), 119

L

Lack of knowledge, 128

Lacuna, 126

le Bars, B., 71

Locus classicus, 121

Loquin, E., 84

$\mathrm{M}$

MacNeil, Lord, 123

Mandate Determination, 147 
Maniruzzaman, A. F. M., 70, 76

Al-Mawardi, 124

Methanex Motonui v. Joseph Spellman \& Ors, 93-94

Mineral policy, 124-126

Mitchell Water Australia Pty Ltd v. McConnell Dowell Constructors (Aust) Pty Ltd, 146-150

Mr Kenneth Davey (Agreement), 151-156

\section{$\mathrm{N}$}

Napoleonic Code, 68

Nathani Steels Ltd. v. Associated Constructions, 162, 165

Nearctic Nickel Mines Inc v. Canadian Royalties Inc, 80

New York Convention, 100, 102, 104, 105

New Zealand, 90, 93

Noble China Inc. v. Lei, 93

N. Radhakrishnan v. Maestro Engineers and Others, 163-165

\section{$\mathrm{O}$}

Onassis Agreement, 120, 121

\section{$\mathrm{P}$}

Pacta sunt servanda, 129-130

Paris, 98

Paris Court of Appeal, 72, 80, 105

Park, W. W., 74

Party autonomy, 99-100

Passive remedy, 88-89, 101, 103, 117

Paulsson, J., 130

Petroleum Development (Trucial Coast) Ltd. v. Sheikh of Abu Dhabi (1951) 18 I.L.R., 122-123

PIL Act, See Swiss Private International Law Act (PIL Act)

Popack v. Lipszyc, 92-93

Predictability, 76

PT First Media TBK, 88

Public policy provisions, 83-84

\section{Q}

Quasi-international treaty, 129

Queensland law, 139-141

\section{$\mathrm{R}$}

Rhone Poulene India Limited (RPIL), 159

Rinehart v. Hancock Prospecting Pty Ltd; Rinehart v. Rinehart, 134-135
April 2007 Deed, 134

Cross-Appellants, 137-138

Deed of Obligation and Release, 134

Full Federal Court, 135-136

High Court, 136-138

Hope Downs Deed, 134, 136, 137

Substantive Claims, 134-136

Validity Claims, 134-136

Robine, E., 75-76

Royal Decree, 120, 121

Rubino-Sammartano, M., 67-68

Russia, 97, 111

RW Health Partnership Pty Ltd v. Lendlease Building Contractors Pty Ltd, 141-145

$S$

Saudi approach, 119

Saudi Arabia, 119-120, See also Aramco case

Saudi Oil Concession Agreement (1933), 125-126

Sauser-Hall, G., 122

Section 16(9) Application, 147

Set-aside mechanism, 89, 92-93, 95, 98-101, 104-112, 114-117

Share Sale Agreement (SSA), 138-139

Singapore International Arbitration Centre (SIAC), 113, 139

Singapore, set-aside proceedings, 87-91, 116-118

contractual waivers, 91-99 arbitration agreement, 114-115

contractual provisions, 115-116

enforcement proceedings, 100-106

exclusions, 106-109, 113-114

grounds of set-aside, 113

international arbitration and foreign parties, 112-113

party autonomy, 99-100

unsuccessful claimant, 109-111

'Smacked of racial superiority,' 126-127

Sornarajah, M. M., 127, 129

Sovereignty, 121

Structural Monitoring Systems Ltd (SMS), 151-156

Structural Monitoring Systems Ltd v. Tulip Bay Pty Ltd, 151-156

Substantive Claims, 134-136

Sukanya Holdings (P) Ltd. v. Jayesh H. Pandya and Anr., 162

Supreme Court Act (SC Act), 110 
Swiss Arbitration Act 1996, 110

Swiss Private International Law Act (PIL Act), 94-97, 115, 116

$\mathrm{T}$

Trade usage, 82-83

Trakman, L., 70, 72, 73

Tulip Bay Pty Ltd v. Structural Monitoring Systems Ltd, 151-156

U

Uncertainty, 76, 77

United Kingdom, 105

United Nations Commission on International Trade Law (UNCITRAL) Model Law, 64-67, 75-79, 82, 83, 85, 88, 90, 92-93

United States, 92

Unpredictability, 76-78

Unsuccessful claimant, 109-111
US Healthcare Food Group Pty Ltd v. Zouky, 139-141

Utility, 73-74

V

Vagenheim, A., 75

Validity Claims, 134-136

Vuillard, E., 75

W

Weinberg, K. S., 76

World Trade Organization (WTO), 120

Y

Yu, Hong-Lin, 66

Z

Zenith Drugs \& Allied Agencies Pvt. Ltd. v. M/s Nicholas Piramal India Ltd., 159-165 